\title{
LA TRANSMISIÓN DE CONOCIMIENTO A TRAVÉS DE LA INFOGRAFÍA DIGITAL
}

\author{
José Luis Valero Sancho \\ (Universidad Autónoma de Barcelona) \\ joseluis.valero@uab.cat
}

\begin{abstract}
Resumen: La infografía digital es un tipo de comunicación muy visual e informativa que refleja asuntos significativos por medio de representaciones figurativas, convenciones abstractas y muy en segundo término por sistemas orales. Es una forma óptima de presentación de mensajes que facilita la comprensión con efectividad similar a la de los textos u otras formas de divulgación establecidas en los medios visuales. Este artículo descubre el incremento de conocimiento que experimentan los estudiantes universitarios ante diversos modelos de infografía digital. Se destaca la diferencia de conocimientos en unas mismas personas antes y después de la interpretación que han realizado.
\end{abstract}

Palabras clave: Infografía, gráficos informáticos, grafismo visual, conocimiento visual.

Abstract: The digital infographics is a very visual type of informative comunication reflecting significant issues through figurative representations, conventions and more abstract oral systems. Is an optimal way of presenting messages that effectively facilitates understanding similar to that of the texts or other forms, established in the visual media. This article reveals the increase of knowledge that students experience with different models of digital graphics. Its highlights the knowledge differencies in the same individuals before and after the interpretation that has been done.

Keywords: Infographics, computer graphics, visual graphics, visual knowledge.

\section{CONTEXTO DE LA INFOGRAFÍA DIGITAL}

a infografía digital es una forma de comunicación visual, informativa y formativa que se reproduce en los medios audiovisuales, normalmente muestra temas significativos de la naturaleza, sucesos acaecidos, fenómenos, conceptos, etc. representados por medios multimediáticos figurativos (dibujos, fotografías o videos), signos convencionales abstractos y sonidos más o menos conocidos por los lectores. Los lenguajes (textos e imágenes) se complementan y superponen constantemente (Alcalá y Santaella, 2004: 111). El propósito con el que se elabora suele ser el de facilitar la comprensión y divulgación de los relatos (descripciones, narraciones o interpretaciones), optimizando y adaptando la forma y recursos al sistema que sea más apropiado para clarificar, desvelar asuntos intrincados, dispersos, etc. y hacerlos fáciles de entender, de agrupar o de mostrar y subrayar en sus tendencias o realidades.

A menudo son sistemas de presentación que explican cuestiones en las que los lenguajes verbales o fotográficos tendrían muchas dificultades para la cons- 
trucción del relato. Las infografías tienen muy buenas cualidades que no pretendemos resaltar aquí, pero sí queremos hacer partícipes a los lectores que son funcionales en algunos aspectos:

1. Por lo que respecta a los relatos periodísticos tienen funciones sintéticas o complementarias especialmente en prensa y prácticamente nunca ocupan un lugar subyugado en los medios digitales periodísticos, donde es un nuevo género infográfico que está destacando por su versatilidad para construir la información integral.

2. En los documentos formativos digitales pueden contener unas ciertas dependencias de los textos, especialmente en los libros digitales y no tanto en los documentos de formación de adultos, enseñanzas técnicas, ocio, etc., donde tienen total autonomía y desvinculación de textos que no se encuentren en su interior.

3. En las divulgaciones comerciales, la infografía digital normalmente se presenta de manera autónoma y autosuficiente, como lo muestran los diversos catálogos comerciales, a mitad de camino entre la venta y la postventa, entre la persuasión y la información del producto ${ }^{20}$.

Podemos decir que los niveles conceptuales de todo lo que contiene la naturaleza necesitan ser presentados para su reconocimiento y entendimiento, por lo que es necesaria una cierta estética que ejerza de puente moderno hacia la ciencia, permitiendo que las expresiones conceptuales se divulguen, entre otras formas, por medio de sensaciones estéticas, como diría el profesor Català (2005: 221).

No quiero dejar pasar la oportunidad de reconocer la posición que tienen al respecto algunos neurocientíficos destacados, quienes afirman que el conocimiento se adquiere de forma sorprendente a partir de lo visual; viendo los conceptos o reconociéndolos se incrementa la posibilidad de "manejarlos" y el empleo de la observación y/o actividad artística mejora el rendimiento de disciplinas no artísticas en el ámbito escolar. Por ello y otras múltiples razones, los psiquiatras están recomendando, empleando y activando esta doble vía de aprendizaje: la actividad visual y artística (Sánchez, 2007). Ambas cualidades son muy propias de la infografía digital.

\section{OBJETO}

El objeto de este estudio es el de poner a prueba la validez de la infografía digital en la transmisión de conocimientos indagando desde distintos tipos de infografía (Valero, 2008a: 631). Deseamos conocer en qué medida tenemos algún

\footnotetext{
${ }^{20}$ Dicho en otros términos, únicamente definimos y tratamos la infografía como documento informativo o formativo, incluso cuando forma parte de una estrategia comercial u otras, no debe entenderse como presentación persuasiva o entretenimiento (Valero, 2008a).
} 
modelo de infografía más interesante que las otras que permita transmitir mejor los conocimientos. Anteriormente hemos realizado pruebas que nos permiten perfilar un modelo ${ }^{21}$, establecer unos límites y elaborar la medición que delate el conocimiento adquirido (Marín, 2007).

La infografía digital es una forma de transmisión de conocimiento, al menos igual de efectiva que la propia de los sistemas lingüísticos literales, aunque se interprete a través de una pantalla. Realizamos una encuesta previa para compararlos dando a leer unos textos a 200 personas con contenidos similares a los que se tenían en una infografía digital y tras su lectura y examen, por el procedimiento que a continuación describiremos para este estudio, obtenían una nota media cercana al 6 sobre 10, aproximadamente la misma que por medio de tres nodos clave representativos de una infografía digital proporcionadas, interpretadas y evaluadas a otras 200 personas distintas. Con ello se demostraba que hay un importante indicio que nos hace suponer que tan válido es el texto como tres nodos de una misma información, ocupando el mismo espacio mediático. Texto y nodos ocupaban la misma extensión, pues los nodos o fotogramas clave se pasaron a papel.

El conocimiento se canaliza a través de los sentidos, que lo clarifican para el entendimiento y que a su vez permiten que lo almacenemos una vez racionalizados. Por eso el proceso requiere tres etapas, a saber: "a) La etapa de captación del estímulo exterior. b) La formación de la imagen perceptiva y c) El reconocimiento del contenido, esto es, la conciencia" (Olivé, 1999).

La medición del conocimiento en las personas es enormemente compleja pero hemos decidido realizar una pequeña prueba de las posibilidades reales que ofrece la infografía para ver mecanismos de crecimiento del conocimiento en los estudiantes universitarios, en su capacidad de asimilación de conocimientos, de temáticas generales diversas en parte desconocidas por ellos, presentadas con tipologías diversas y no necesariamente periodísticas.

\section{MODELO DE INVESTIGACIÓN}

El modelo de investigación lo comenzamos desde la delimitación de la investigación:

1. Todas las preguntas de cada uno de los ocho cuestionarios son parecidas pero no exactamente iguales.

2. No se permite presentar un tema propio de un área de conocimiento situado en la carrera que el estudiante realiza.

\footnotetext{
${ }^{21}$ Doy las gracias a algunos estudiantes del curso 2008-2009 de doctorado del Departamento de Comunicación Audiovisual y de Publicidad titulado "El relato en la infografía digital" que han colaborado desinteresadamente en la realización de la encuesta: ARGUETA ARGUETA, Yovanny Francisco; PUERTAS GRAU, Helena; REINA JIMÉNEZ, Rafael; RODRIGUES DOS SANTOS, Joao Paulo; SOLA MORALES, Salomé; CASTILLO BETANCOURT, Camilo y LUNA RASSA, María Fernanda.
} 
3. No se eligen infografías de temas de interés mediático actual (si aparecieron en los medios, tienen un mínimo de un año de antigüedad) para no preguntar sobre temas de actualidad muy reciente.

4. No se eligen infografías digitales de elmundo.es y elpais.com pero pueden ser de cualquier otro medio, periodístico $0 \mathrm{no}^{22}$.

5. Se realiza interpretando la infografía en la pantalla del ordenador conectado a la red, nunca en hojas de papel impreso.

6. No se informa de nada de lo que vendrá después para no incurrir en factores de distorsión o encubrimiento.

7. Ninguna infografía seleccionada contiene más de 16 nodos para delimitar su extensión.

8. Los sujetos experimentales son estudiantes matriculados en la UAB pues no pretendemos analizar nada relacionado con las características sociométricas de ningún tipo de sociedad, sino fijar como constantes a los intérpretes ${ }^{23}$.

Son tres los modelos de infografía digital que aparecen a partir del primer nodo de portada:

1. Modelo funcional sin botones ${ }^{24}$, de estructura totalmente horizontal como podría ser cualquier clase de mapa con ventanas para el detalle, se le ha llamado también de "supermercado".

2. Modelo lineal de estructura vertical sin botones que permite avanzar o retroceder por medio de botones de navegación o sistemas de dial, también denominados "de edificio".

3. El modelo más empleado es el de tipo lineo-funcional que tras la portada tiene botones para poder optar por varias entradas a diversos aspectos (serían como apartados, igual que se realiza en los artículos escritos e impresos) del tema que trata la infografía. Tras el botón que nos sitúa en una de las líneas de contenido empleando el modelo lineal (Valero, 2003b) que puede tener ventanas al tamaño de los grafismos o más grandes con una cierta navegación horizontal.

\footnotetext{
${ }^{22}$ Hemos desechado las infografías de elpais.com y elmundo.es debido a su fácil acceso y estructuras de presentación muy uniformes y estándares, que todo el mundo profesional español toma como modelos de referencia. Además no se pone como condición que sean periodísticas, ya que pueden ser del resto del universo infográfico con estas dos excepciones. También es interesante apreciar que de esta forma se intentan buscar temáticas ilimitadas espacialmente para el interés de los estudiantes de la UAB, pues pocos medios españoles de comunicación contienen infografías con esos grados de elaboración.

${ }^{23}$ Término empleado por Pericot (2002: 24) para referirse al sujeto para el que la imagen tiene función sígnica, distinguiendo al "interpretante" como el efecto o vehículo sígnico que actúa sobre el "intérprete".

${ }^{24}$ Entendemos por botón el pequeño espacio informático que acciona una función al pasar o pulsar sobre él con el ratón de ordenador.
} 
Por otro lado, si convenimos con el Diccionario de la Real Academia de la Lengua que nodo es "cada uno de los puntos que permanecen fijos en un cuerpo vibrante. En una cuerda vibrante son siempre nodos los extremos, y puede haber varios nodos intermedios", nosotros empleamos este término para referirnos, en sentido figurado, a la cantidad de "clics" "fotogramas clave o key frames", según el concepto que toman programas como Flash (CS3), Affter Effects (CS3) - 3Dmax2008 que ponemos para delimitar las infografías seleccionadas, de tal manera que se traten de documentos cortos pero que se puedan abarcar en el tiempo prefijado para la interpretación. Es decir, las infografías pueden tener una extensión máxima de 16 nodos consecutivos en una infografía funcional o lineal, que a su vez se corresponden a una estructura lineo-funcional media de cuatro botones con cuatro nodos cada uno, con las variantes que se tengan 25 .

Por tipología entendemos el "estudio y clasificación de tipos que se practica en diversas ciencias" (Valero, 2008a: 632-636), es decir, la clasificación propia de la infografía de cuatro conjuntos de muestras: dos comparativas, dos ubicativas, dos escénicas y dos documentales que componen las ocho infografías seleccionadas, de las que se preguntaron diez cuestiones a quince estudiantes.

Tipología y direcciones de las infografías elegidas:

1. Comparativa: Sida: http://www.abc.es/informacion/graficosflash/sociedad sida.asp

2. Comparativa: Expectativas de vida http://www.gapminder.org/

3. Documental: Consecuencias de la deshidratación: http://consumer.es/infografias/

4. Documental: Anisakis http://consumer.es/infografias/

5. Escénica: Atentado a un edificio http://www.clarin.com/diario/especiales/infos/graficos/if_amia.html

6. Escénica: El hundimiento del Belgrano http://www.clarin.com/diario/2007/04/02/conexiones/malvinas07.html

7. Ubicativa: San Fermín http://www.diariodenavarra.es/graficosFlash/index. asp?id=sanfermin 2006

8. Ubicativa: La Meca http://www.hoy.com.ec/infografias/espanol/animacion/ MeccaES1812/index1.html

¿Cómo sabemos si una infografía es comparativa, ubicativa, escénica o documental? Atendiendo al tipo que tenga cada nodo y detectando el dominio tipológico mayoritario. Por ejemplo, 2 nodos comparativos, 3 nodos escénicos y 7 nodos ubicativos nos darían una infografía predominantemente ubicativa que

\footnotetext{
${ }^{25}$ Si tomáramos Google Earth como infografía, no podríamos emplearlo en esta investigación pues se sobrepasarían enseguida los 16 nodos.
} 
podría emplearse en la encuesta dado que tiene un número total de 12 nodos que obviamente no llega a los 16 marcados como límite.

Interpretación e interpretar personalmente consiste en concebir, ordenar o expresar de un modo personal la realidad ${ }^{26}$. Los estudiantes se someten a la interpretación de la infografía que les toque, por tanto se les sitúa ante la portada de la infografía y buscan con libertad en el ordenador, en el documento en red, de lectura lineal como la literal, anárquica como la propia de las imágenes o incluso ambas cosas: unas veces juntas como en los mapas con textos ${ }^{27}$ y otras por separado como en las fotografías titulares o dibujos.

Los cuestionarios contienen preguntas genéricas adaptadas a cada una de las ocho infografías, las cuales se responden de forma concreta, pero cada infografía tiene un cierto acondicionamiento de la forma de presentar la pregunta, motivado por su temática. El estudiante, de forma anticipada, rellenó un cuestionario sobre el tema del que trata la infografía que se le dio a interpretar, para así detectar el conocimiento que tenía procedente de otras formas anteriores de incremento de conocimiento. En este momento únicamente se le mostró lo que contiene la portada infográfica apoyada con unos breves comentarios no explicativos del encuestador. Por último y sin mediar tiempo alguno se les dejó ante la portada en el ordenador y se les dieron cinco minutos de interpretación de la infografía, tras los que rellenaron de nuevo un cuestionario que, para su sorpresa, resultaba ser el mismo del principio.

Entendemos que el conocimiento es la averiguación por el ejercicio de las facultades intelectuales del ser humano de la naturaleza, cualidades y relaciones de las cosas. Por ello, el incremento de conocimiento debía aparecer tras obtener la diferencia entre el antes y el después de la consulta. Empleamos para su medición el sistema tradicional de examen de test, en el que cada pregunta acertada tiene un valor de 1/10 (sólo una de las cuatro contestaciones es válida). Este tipo de examen se emplea habitualmente en el ámbito universitario clásico y aunque no es un sistema óptimo de medición que permite la posterior evaluación, sí se puede decir que es muy funcional, rápido y preciso para medir o evaluar el conocimiento que tienen los estudiantes de los objetos o asuntos que contiene la naturaleza ${ }^{28}$, cualidades o relaciones de las cosas, etc.

Como el propio cuestionario brinda la posibilidad de que una de las cuatro contestaciones sea cierta, puede por ello, aunque apenas ha ocurrido en este caso, dar un valor inferior tras interpretar la infografía especialmente en alguna de

\footnotetext{
${ }^{26}$ Como en infografía se lee pero también se miran las imágenes, hemos optado por añadir el concepto de interpretación que permite la lectura, audición y visualización en doble sentido, pues visualidad y teleespectación son dos aspectos distintos de una misma manera de ver, no leyendo (entendiendo que sólo se lee lo literal y gracias a una alfabetización escolar).

${ }^{27}$ Ver el concepto de infograma entendido como unidad compleja de los elementos menores de la infografía (Valero, 2008b).

${ }^{28}$ Material o inmaterial, pasada, presente o futura, hechos fortuitos o actos de seres vivos, en general se pueden describir con el concepto genérico y ambiguo de "cosas".
} 
las contestaciones realizadas y acertadas inicialmente al azar, pero contestadas después erróneamente, que no dejan de ser un cierto factor encubridor.

Por último y por medio de las tablas de recogida conjuntas podemos obtener valores totales máximos y mínimos, generar fórmulas y comparar datos de indicaciones locales o individuales.

También depende del modelo que se presente al sujeto experimental y por ello buscamos destacar la presentación tipológica que más éxito tenga en el incremento de conocimiento. Según el tipo de infografía elegido, empleamos el análisis indirecto de la comparación de coeficientes de correlación de Pearson ${ }^{29}$, y especialmente el directo de los valores totales variables, para resaltar en qué medida la variable independiente crece más cuando el estudiante sabe más del tema o lo que es lo mismo, cuando obtiene una más alta nota inicial.

Somos conscientes de la supuesta visión limitada que estos datos pueden aportar en sus resultados y comparaciones y por ello tomamos con reservas los resultados, pero también con el ánimo de que contribuya a la elaboración de una forma de medir el grado de conocimiento adquirido por un producto multilineal y desordenado en cuanto a su interpretación por las personas. No pretendemos sino hacer una prueba indicial que abra el camino hacia horizontes científicos efectivos y mejor elaborados a nivel estadístico.

\section{RESULTADOS CUANTITATIVOS GENERALES}

El gráfico 1 muestra de manera general el crecimiento total de conocimiento medido con 8 infografías, 15 encuestados y 10 preguntas por encuestado que han sido contestadas dos veces, por tanto tenemos 2.400 contestaciones totales. A pesar del alto conocimiento previo que tienen los estudiantes respecto a ocho temáticas variadas ${ }^{30}$, la infografía incrementa el conocimiento en un $86 \%$. Entendemos que puede ser el valor de una prueba que no lo mejorarían probablemente otro tipo de aportaciones comunicativas visuales. Esa puede ser una comparación posterior a este estudio.

\footnotetext{
${ }^{29}$ Tomado de los conceptos generales de estadística.

${ }^{30}$ Que hacen suponer que si contestaran a todo, de manera correcta, invalidaría nuestra forma de medir, puesto que no tendríamos recorrido para incrementar la cuantificación con la que medimos el conocimiento.
} 
Gráfico 1. Aumento del conocimiento total adquirido por parte de los 125 estudiantes.

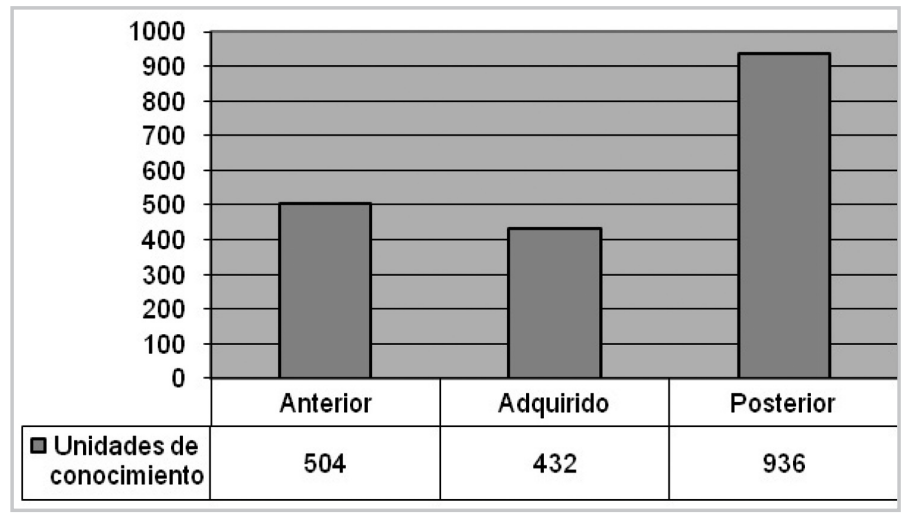

Fuente: Elaboración propia.

También es cierto que si los estudiantes supieran muy poco de los temas que tratan las infografías podrían tener una buena oportunidad de entender cuestiones que no conocen y quizá las notas tendrían un incremento de valor adquirido.

Entre la matriz formada por la nota anterior y la posterior hay un cierto grado de correlación positiva gracias a la influencia de la interpretación infográfica, ya que es relativamente correlativa con un indicador de 0,47 del coeficiente de correlación de Pearson que se sitúa entre de un intervalo total de correlación de +1 y de no correlación en -1.

Gráfico 2. Incremento de conocimiento por temáticas.

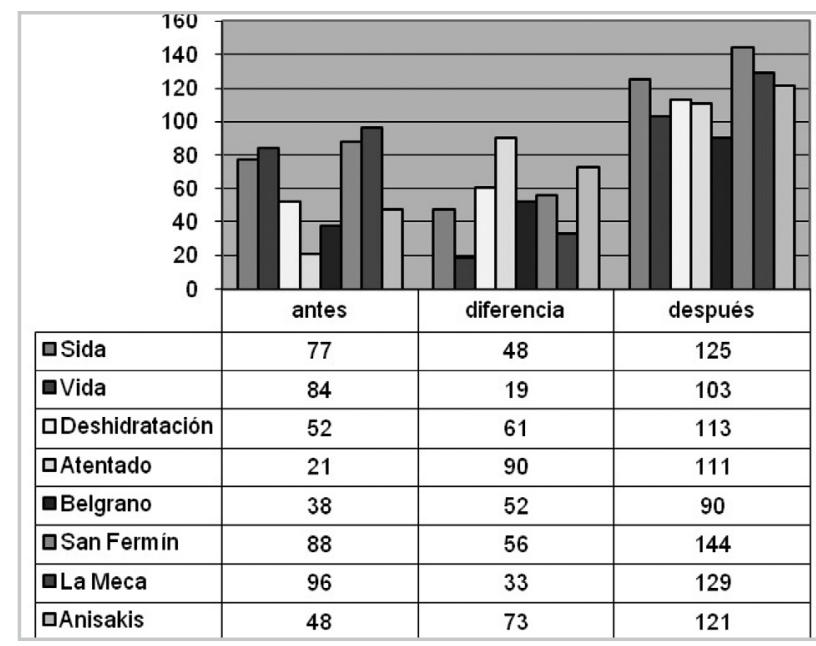

Fuente: Elaboración propia. 
En el gráfico 2 Se aprecia que hay temáticas que el sujeto experimental intérprete conoce bien y por esa razón la infografía no le aporta porcentualmente mucho conocimiento, como en la infografía "Expectativas de vida". Sin embargo, se puede observar que el mayor valor de conocimiento se corresponde con las infografías "La Meca" y "San Fermín", ambas cuestiones presentadas tipológicamente de forma ubicativa, pero también son temas de grandes concentraciones humanas, muy del interés de la juventud universitaria, aunque en este caso de claro signo distinto una y otra.

En las que se desconoce bastante el tema su crecimiento es muy importante, se observa que hay un desconocimiento inicial mayor de las consideradas más periodísticas, como es el caso del atentado a un edificio o el hundimiento del Belgrano, en ambas se muestra un importante crecimiento del conocimiento.

Por otro lado, en el gráfico 3 llama la atención en las preguntas una cierta uniformidad entre los conocimientos anteriores y finales. Sin embargo, se aprecia que quizá algunos temas o preguntas responden a cuestiones con mayor acierto previo que otras, tal sería el caso de las relacionadas con el qué, quién, dónde, cuándo y cómo, puesto que todas ellas superan los 100 puntos de conocimiento previo.

La uniformidad conseguida a pesar de que la adaptación concreta de las preguntas genéricas ha sido problemática, muestra un grado de aceptación de la pregunta, de manera regular en los ocho casos. No se ha visto en las encuestas individuales prácticamente ningún problema con las contestaciones o las aclaraciones conceptuales.

Gráfico 3. Resultados de la encuesta con preguntas genéricas adaptadas a cada temática.

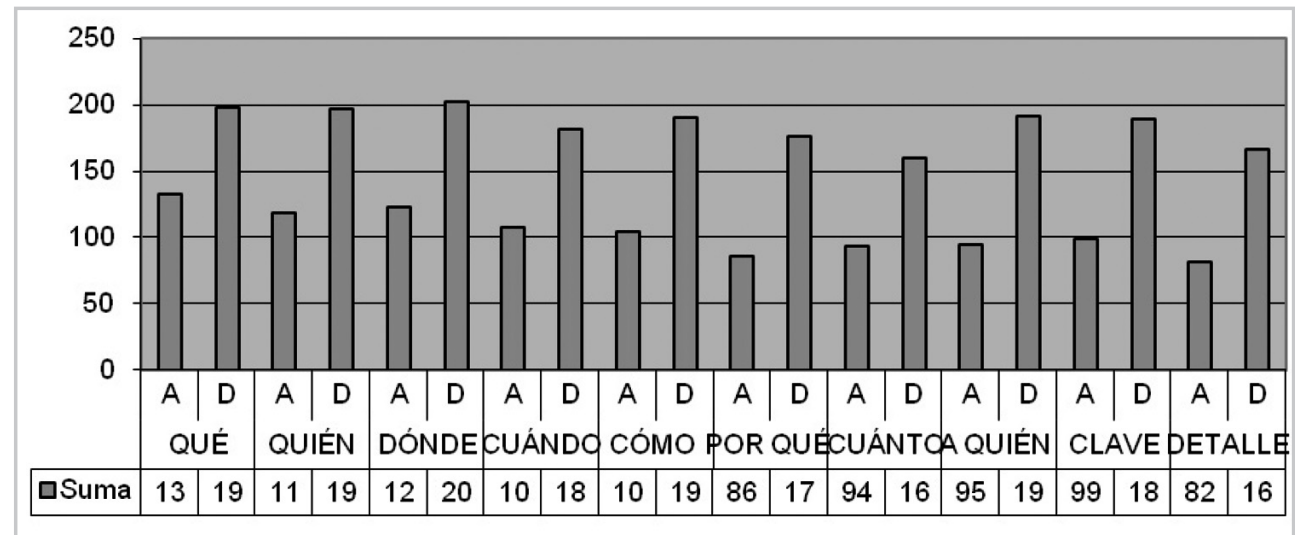

Fuente: Elaboración propia ${ }^{31}$.

\footnotetext{
${ }^{31} \mathrm{~A}$ significa consulta anterior a la exhibición de la infografía y $\mathrm{D}$ significa consulta posterior.
} 
Por lo que respecta a la tipología, en el gráfico 4 se aprecian unos valores de crecimiento espectacular del conocimiento en el caso de las infografías escénicas, probablemente motivado por los dibujos más propiamente figurativos que los de otros tipos que, al ser más abstractos, tienen un menor grado de familiaridad en las interpretaciones.

Gráfico 4. Resultados tipológicos comparados.

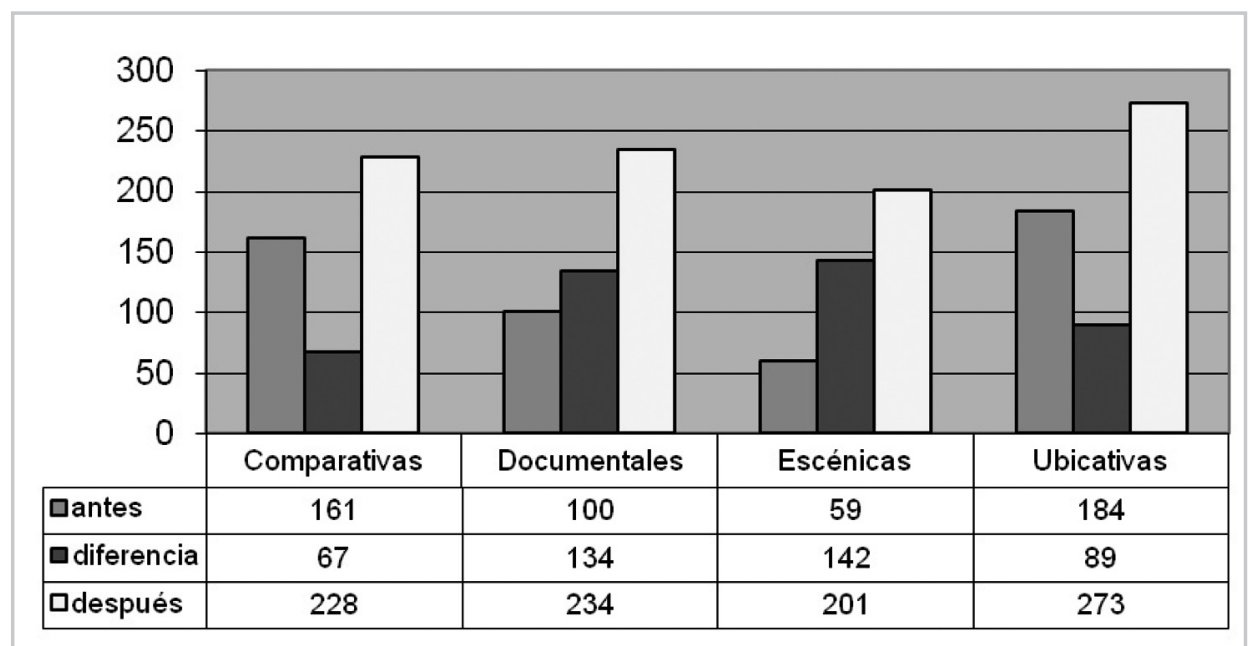

Fuente: Elaboración propia.

En este sentido llama la atención el poco crecimiento de conocimiento que tienen los tipos mayoritariamente elaborados con gráficos (comparativas) y los elaborados mayoritariamente con mapas, planos o microplanos (ubicativas) frente a los documentales y escénicos que admiten otro tipo de presentaciones más originales y figurativos (a pesar de que un mapa es figurativo, no lo es en el sentido de la escala de proximidad que tienen los objetos más cercanos).

Como reflexión final a estos gráficos, parece que siempre es mayor conocimiento que los sujetos experimentales intérpretes tienen que el que les proporciona la infografía, lo cual da una cierta idea de la importante formación universitaria que poseen y que quizá sería interesante compararla con la de otros sujetos de características distintas ante temáticas iguales.

\section{CONCLUSIONES GENERALES}

Los estudiantes cuando interpretan algunas de las temáticas de una infografía digital, de las que tienen unos ciertos conceptos gracias al trasvase de otras 
fuentes informativas o culturales, aumentan y clarifican considerablemente su nivel de conocimientos sobre esas cuestiones.

Es una forma de presentación idónea para dar una visión del pasado, escenificar el presente e incluso imaginar el futuro. Es una herramienta importante para que los intérpretes consultados accedan a documentos visuales bien elaborados documentalmente y presentados visualmente con la interactividad y el tiempo necesarios para su consulta.

La infografía digital tiene un importante poder estético de captación de la atención dada su fuerte componente visual. En ella se presentan diversos y originales recursos estéticos y visuales, sin sensación de cúmulo de datos, que permiten el juego de la imaginación a través de comparaciones inesperadas, metáforas originales, etc. Además de contar con ese poderoso instrumento tipográfico siempre necesario en pequeñas dosis, con el tiempo únicamente limitado por el intérprete para su lectura.

Es cercana al ser humano moderno, pues permite al intérprete en su interactividad navegadora ser testigo directo en sus indagaciones, con un manejo de mandos que en algo se parece a los que emplea en su vida diaria y costumbres cotidianas, adiestradas en el manejo de teléfonos, ordenadores o electrodomésticos, de manera que fácilmente asume su funcionamiento, aunque sus botones o símbolos de manejo puedan tener un cierto componente original.

Podemos decir que el conocimiento se puede obtener por diversos medios, que en el caso del sistema visual puede ser la lectura tipográfica, sistema que ha facilitado la adquisición de conocimiento desde el Renacimiento hasta nuestros días. Sin embargo hay alternativas en la actualidad como la que se presenta como infografía digital que permite hacer realidad una nueva forma de adquisición de conocimiento, como en los resultados se ha demostrado.

El intérprete, tras observar la infografía digital a través de una pantalla con teclado, interactúa con el documento digital, motivado por el interés que le despierta el documento presentado, con ciertas dosis estéticas modernas y penetra en el desarrollo temático de lo que se presenta, viendo y leyendo hasta comprenderlo. Este estudio muestra y desvela los conocimientos adquiridos al evaluarlo.

En definitiva, y como conclusión final, el conocimiento no debemos entenderlo como una actividad humana consciente e inmediata, sino como un conjunto interpretativo en el marco interactivo, especialmente dinámico, libre y especializado en temáticas apropiadas (como se ha visto en los resultados), cuando se observan infografías. Los relatos visuales se presentan para hablarle pragmáticamente al intérprete (Pericot, 2002: 27) y para ello los preparan expertos ${ }^{32}$ de forma concien-

\footnotetext{
${ }^{32}$ EI postgrado de la UAB titulado "Grafismo informativo y documental", dirigido por quien suscribe, tiene el honor de contar con los infógrafos más importantes de Catalunya como son Jordi Català o Jaume Serra y algunas de las figuras profesionales más destacadas de la infografía digital como Alberto Cairo, Dolors Pou, etc.
} 
zuda. Éste y otros estudios similares permiten conocer las condiciones adecuadas que satisfacen la transmisión de conocimiento.

\section{REFERENCIAS BIBLIOGRÁFICAS}

BERTIN, J. (1988): La Gráfica y el Tratamiento Gráfico de la Información. Madrid: Taurus Comunicación.

CABRERA, M. A. (2000): La prensa online. Barcelona: CIMS.

CAIRO, A. (2008): Infografía 2.0. Madrid: Alamunt.

CARD, S.; MACKINLAY, J.; Y SHNEIDERMAN, B. (1999): Readings in Information Visualization: Using Vision To Think. San Francisco: Morgan Kaufmann Publishers.

CATALÀ, J. M. (2005): La imagen compleja. Barcelona: Servei de Publicacions de la UAB.

CONTRERAS, F. R. (2000): Nuevas fronteras de la infografía. Análisis de la imagen por ordenador. Sevilla: Mergablum.

De PABLOS, J. M.: "Siempre ha habido infografía". Revista Latina de Comunicación Social, [en línea]. 1998. [Consulta: 5 octubre 2008]. <http://www.ull.es/publicaciones/latina/ a/88depablos.htm>

GRAU, O. (2003): Virtual Art. From/llusion to Immersion. Cambridge: The MIT Press.

HARROWER, T. (2002): Newspaper Designer's Handbook. McGraw-Hill.

HOLMES, N. (2005): Wordless Diagrams. Bloomsbury.

IVINS, W. (1975): Imagen impresa y conocimiento. Análisis de la imagen prefotográfica. Barcelona: Gustavo Gili.

MANZINI, E. (1992): Artefactos: Hacia una nueva ecología del ambiente artificial. Madrid: Celeste Ediciones.

MARÍN, B. (2007): La Infografía digital. Un modelo de transmisión alternativa de conocimiento. Trabajo de Investigación inédito, Universidad Autónoma de Barcelona.

MARTíNEZ-SALANOVA, E.: "La infografía, un instrumento para el análisis". Aula Creativa, [en línea]. 2004. [Consulta: 4 septiembre 2007].

$<$ http://www.uhu.es/cine.educacion/cineyeducacion/salanova.htm>

MINERVINI, M. A. (2005): "La infografía, como recurso didáctico". Revista Latina de Comunicación Social, [en línea]. 2005. [Consulta: 10 octubre 2008]. <http://www.ull.es/publicaciones/latina/200506minervini.pdf>

OLIVÉ, A. (1999): "Aproximación al Conocimiento Humano: Un problema y sus consecuencias". A Parte Rei. [en línea]. 1999. [Consulta: 4 septiembre 2008]. <http://serbal.pntic.mec. es/ cmunoz11/olive.html>

PERICOT, J. (2002): Mostrar para decir. Barcelona: Servei de Publicacions de la UAB y otras.

SÁNCHEZ, X. "Arte visual en la escuela". La Vanguardia, 12 de octubre de 2007. 
VALERO, J. L.: El relato en la infografía digital. En DÍAZ, J.; y SALAVERRIA, R. (2003): Manual de Redacción Ciberperiodística. Barcelona: Ariel, pp. 555-589.

- (2008a): "Tipología del grafismo informativo". Estudios sobre el mensaje periodístico, $\mathrm{n}^{\circ}$ 14 (octubre 2008), pp. 631-648.

- (2008b): "La infografía digital en el ciberperiodismo". Revista Latina de Comunicación Social. [en línea]. 2008. [Consulta: 1 enero 2009] <http://www.ull.es/publicaciones/latina/08/42 79965 Bellaterra/forma.html>

\section{Breve semblanza biográfica del autor}

José Luis Valero Sancho es Doctor en Comunicación Audiovisual. Desde 2001 es profesor titular de Comunicación Audiovisual y de Publicidad de la UAB. Miembro del grupo de investigación GRISS de la UAB y del comité de administración de la revista Textual \& Visual Media. Está especializado en temas de desarrollo visual en prensa, internet y televisión. Es director del máster de "Artes e industrias gráficas" y del postgrado de "Grafismo informativo y documental", ambos de la UAB.

(Recibido el 04-04-2009, aceptado el 03-06-2009) 